\title{
Rare Giant Upper Lip Epidermal Cyst in a Patient Wearing a Denture
}

\author{
Jong Chan Kim ${ }^{1}$, \\ In Pyo Hong ${ }^{2}$ \\ ${ }^{1}$ Department of Plastic and Reconstructive \\ Surgery, National Medical Center, Seoul; \\ ${ }^{2}$ Department of Plastic and Reconstructive \\ Surgery, Eulji University Hospital, Eulji \\ University School of Medicine, Daejeon, \\ Korea
}

No potential conflict of interest relevant to this article was reported.

\begin{abstract}
Epidermal cysts are intradermal or subcutaneous cystic tumors that frequently occur in the face, scalp, neck, and body trunk. Acquired cases of epidermal cyst commonly occur as a result of various surgical operations, chronic irritation, or trauma, all of which may trigger the occurrence of the invagination of squamous epithelium. A 57-year-old man presented with a palpable mass $7 \mathrm{~cm} \times 2 \mathrm{~cm}$ in size in the upper lip. The patient had a 3-year history of wearing a denture to restore missing bilateral maxillary central and lateral incisors, accompanied by inflammatory findings on the buccal mucosa due to chronic lip irritation. The resected oval-shaped cyst had a size of $5.5 \mathrm{~cm} \times 3.0 \mathrm{~cm} \times 2.5 \mathrm{~cm}$, and it was an encapsulated mass with a well-defined margin. The histopathology was typical of epidermal cyst. This case of a rare giant upper lip epidermal cyst in a patient wearing a denture may be of interest to clinicians.
\end{abstract}

Keywords: Giant epidermal cyst / Dentures / Chronic irritation

\section{INTRODUCTION}

Epidermal cysts are a benign skin disease with a relatively high prevalence. They may occur in congenital or acquired forms. They frequently present as intradermal or subcutaneous cystic tumors in the face, scalp, neck, and body trunk [1]. According to a review of the literature, no definition of the giant epidermal cyst has been established. Because the size of epidermal cysts normally varies from 1 to $5 \mathrm{~cm}$ in diameter, it has generally been accepted that the giant epidermal cyst is defined as an epidermal cyst $\geq 5$ $\mathrm{cm}$ in diameter [2]. In particular, acquired cases of epidermal cysts commonly occur as a result of a surgical procedure, chronic irritation, or trauma, all of which may trigger the occurrence of the invagination of squamous epithelium. Epidermal cysts occurring in the lips and philtrum show a clinical presentation that is similar to those arising from other infections or injections of foreign body

\section{Correspondence: In Pyo Hong}

Department of Plastic and Reconstructive Surgery, Eulji University Hospital, Eulji University School of Medicine, 95 Dunsanseo-ro, Seo-gu, Daejeon 35233, Korea

E-mail: nmcps@unitel.co.kr

Received June 8, 2016 / Revised November 29, 2016 / Accepted November 29, 2016 materials such as fillers. This poses a differential diagnostic challenge for clinicians. To date, many authors have described cases of acquired epidermal cyst. However, no case of acquired giant upper lip epidermal cyst in a patient wearing a denture has previously been reported. Here, we report our case with a review of the literature.

\section{CASE REPORT}

A 57-year-old man visited us with a chief complaint of a mass occurring in both the superior lip and the philtrum (Fig. 1). The patient had a 3-year-history of wearing a denture to restore missing bilateral maxillary central and lateral incisors, accompanied by inflammatory findings on the buccal mucosa due to chronic lip irritation. The patient did not perceive the presence of a mass before wearing a denture. Several months thereafter, however, the patient perceived a slowly growing mass. Three months before visiting us, the patient complained of erythema and pain. On our physical examinations, the patient had a palpable mass of $7.0 \mathrm{~cm} \times$ $2.0 \mathrm{~cm}$ in the upper lip concurrently with mild erythema and ten- 


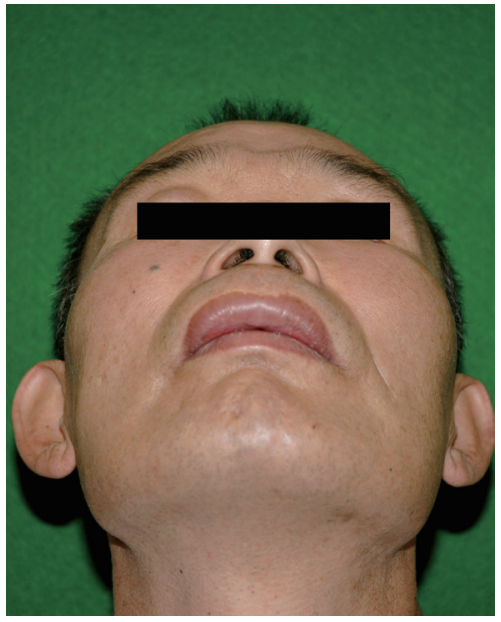

Fig. 1. Preoperative view. The mass occurring in both superior lips and philtrum.

derness. There was a puncture on its surface, where no secretory material was present. We assumed that the patient presented with inflammatory findings due to the denture, and therefore planned to perform a resection of the mass under local anesthesia. Preventing the rupture of the membrane surrounding the mass, we dissected the adhesion between the mass and the adjacent cystic tissue while minimizing the damage to the adjacent anatomical structures using a mosquito clamp (Fig. 2). Its interior contents were composed of sticky sebum with a bad odor. On the histopathology, the cystic wall was composed of a multilayer of epithelial cells containing keratohyaline granules. These findings are suggestive of a typical epidermal cyst (Fig. 3A). Many monocytes, lymphocytes, and granulomas were found on histopathology, which was suggestive of chronic inflammation, concurrently with hyperplasia and keratinization of the mucosal cells (Fig. 3B).

\section{DISCUSSION}

According to a review of the literature, epidermal cysts represent $1.6 \%-6.9 \%$ of the cysts affecting the head and neck, and less than $0.01 \%$ of cases of oral cysts [3]. Various theories have been proposed regarding the origin of the dermoid/epidermoid cyst. Among the theories, previous surgical or traumatic events may cause traumatic implantation of epithelial cells into deeper tissues,

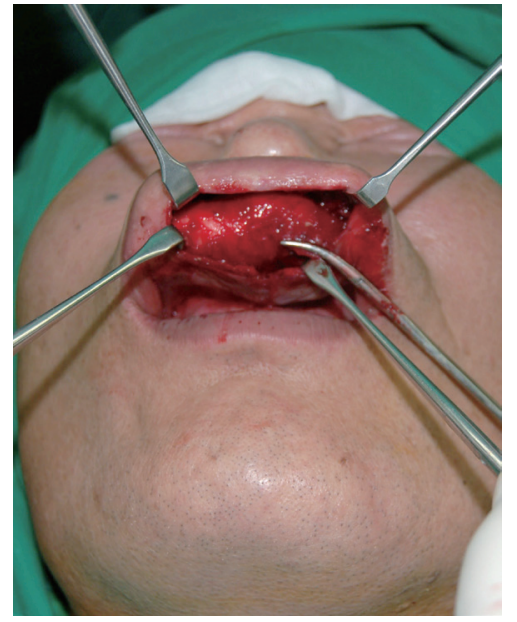

Fig. 2. The dissected oval-shaped giant mass with minimizing the damages to the adjacent anatomical structures.
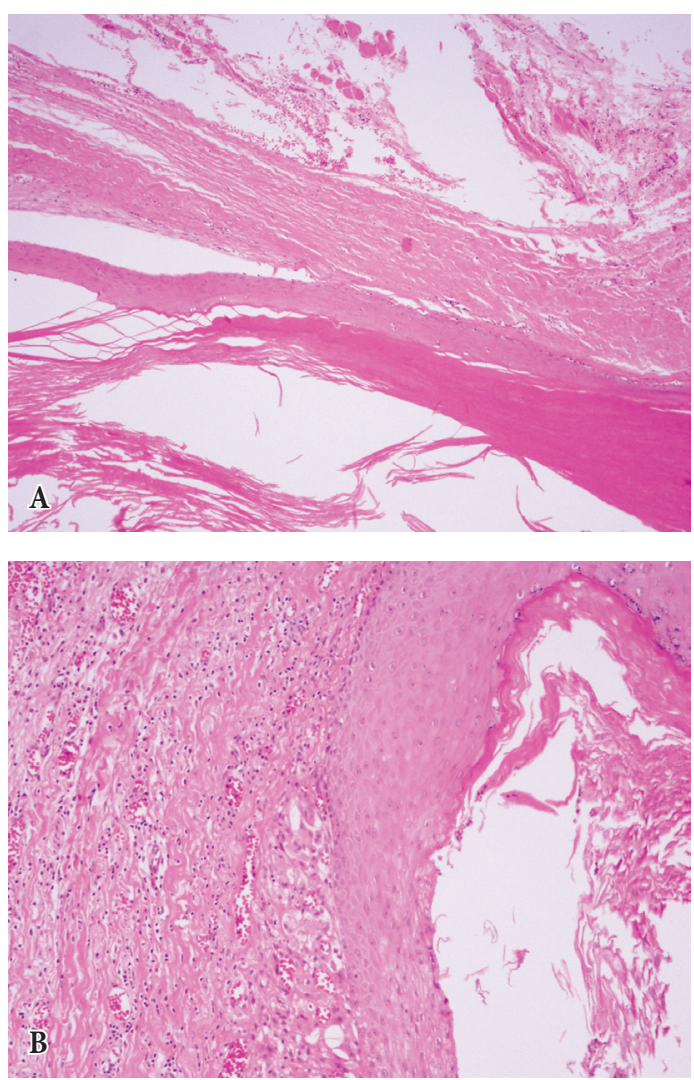

Fig. 3. (A) On histopathology, the cystic wall is composed of a multilayer of epithelial cells containing keratohyaline granules, accompanied by chronic inflammation and keratinization of mucosa and epidermoid cyst $(\mathrm{H} \& \mathrm{E}, \times 100)$. (B) There are many monocytes, lymphocytes and granuloma, suggestive of chronic inflammation, concurrently with hyperplasia and keratinization of mucosal cells $(\mathrm{H} \& \mathrm{E}, \times 200)$. 
which may give rise to an implantation dermoid [4]. It is worth noting that our case occurred in an unusual location, the upper lip, accompanied with chronic irritation due to the denture. The patient had no notable traumatic findings on the history before wearing a denture. Thereafter, based on a disease history, surgical findings, and pathologic results, we reasonably believe that this giant epidermal cyst resulted from chronic inflammation due to the denture. In the current case, the giant epidermal cyst was enlarged from a small epidermal one.

In conclusion, our case indicates that it worth educating patients on how to use dentures in order to prevent the progression of irritation to giant epidermal cyst [5]. We experienced a rare giant upper lip epidermal cyst in a patient wearing a denture, which may be of interest to clinicians. Here, we report our case with a re- view of the literature.

\section{REFERENCES}

1. Lever WF, Schaumburg-Lever G. Histopathology of the skin. 7th ed. Philadelphia: Lippincott; 1990.

2. Rios Arizpe S, Ocampo Candiani J. Giant epidermoid cyst: clinical aspect and surgical management. J Dermatol Surg Oncol 1986;12:7346.

3. Calderon S, Kaplan I. Concomitant sublingual and submental epidermoid cysts: a case report. J Oral Maxillofac Surg 1993;51:790-2.

4. De Ponte FS, Brunelli A, Marchetti E, Bottini DJ. Sublingual epidermoid cyst. J Craniofac Surg 2002;13:308-10.

5. Bilhan H, Geckili O, Ergin S, Erdogan O, Ates G. Evaluation of satisfaction and complications in patients with existing complete dentures. JOral Sci 2013;55:29-37. 